\title{
Melatonin versus chloral hydrate as the sedating agent in performing electroencephalogram in paediatric patients
}

\author{
C.L. Yuen ${ }^{\text {a,* }}$, W.W. Cherk ${ }^{\text {a }}$, T.H. Fung ${ }^{\text {a }}$, C.S. Ho ${ }^{\text {a }}$, K.K. Chan ${ }^{\text {a }}$, Y.W. Yu ${ }^{\text {b }}$ \\ ${ }^{a}$ Department of Paediatrics, Kwong Wah Hospital, Hong Kong Special Administrative Region \\ ${ }^{\mathrm{b}}$ Electrodiagnostic Unit, Kwong Wah Hospital, Hong Kong Special Administrative Region
}

\section{A R T I C L E I N F O}

\section{Article history:}

Received 21 August 2016

Accepted 21 November 2016

Available online 3 January 2017

\section{Keywords:}

EEG

Melatonin

Chloral hydrate

Sedation

Paediatric

\begin{abstract}
A B S T R A C T
Electroencephalography (EEG) is a valuable tool in the diagnosis of epilepsy. The attainment of a high quality EEG requires patient's co-operation which is particularly difficult in children. Chloral hydrate has been used as a sedating agent in EEGs but it has potential serious adverse effects and anti-epileptic activity. Melatonin is used increasingly in different investigations as a safe alternative. Our study is to compare their effectiveness as sedating agents in performing EEGs and the detection rate of abnormal EEGs. This is a retrospective study performed in a regional hospital in Hong Kong. One hundred and ninety two EEG studies were included from December 2010 to July 2014. One hundred and two children were given chloral hydrate $(50 \mathrm{mg} / \mathrm{Kg})$ in the first half of the period and 90 children were given melatonin ( $3 \mathrm{mg}$ for $=<5$ years or $6 \mathrm{mg}$ for $>=5$ year) in the later half. The two groups are compared with Pearson's Chi-squared test with Yates' continuity correction. The successful rate in sedation was similar between the two groups while the pick up rate of abnormal EEGs was $52.56 \%$ in the melatonin group and $21.57 \%$ in the chloral hydrate group $(p<0.05)$. Subgroup analysis among patients with epilepsy or mental retardation and intellectual disability shared same findings with higher detection rate of abnormal EEGs in the melatonin group. No side effect was documented in the study. Compare with chloral hydrate, melatonin is a safe and effective alternative and probably has less interference with the electrographic activity.
\end{abstract}

(C) 2016 Published by Elsevier, a division of RELX India, Pvt. Ltd on behalf of Indian Epilepsy Society.

\section{Introduction}

A high quality EEG requires cooperation of the individual which is particularly difficult for paediatric populations and patients with intellectual problems. Behavioural trainings and sleep deprivation can work in selected cases but they are time consuming and require immense input from both the carers and medical staff. ${ }^{1}$ Chloral hydrate has been widely used as an effective sedating agent in EEGs in adult and paediatric populations. However, it has the drawback of altering the sleep architectures, potential antiepileptic effects as well as the rare occurrence of serious side effects due to deep sedation. Therefore the exploration of an alternative sedating agents is warranted. Melatonin (N-acetyl-5methoxytryptamine), a natural hormone, is found effective and safe as a sedation in different diagnostic procedures. ${ }^{2}$ The aim of this study is to compare the effectiveness of melatonin against

\footnotetext{
* Corresponding author.

E-mail address: clyuen@icloud.com (C.L. Yuen).
}

chloral hydrate as a sedating agent for EEGs and would there be any difference in the detection rate of abnormal electrographic discharges.

\section{Methods}

This study is a retrospective study of patients requiring sedation for routine EEGs in the department of paediatrics of a regional hospital in Hong Kong from December 2010 to July 2014. There was a change in sedation protocol in the middle of this period in October 2012 from chloral hydrate to melatonin as the primary sedative agent. There was no change in all other details of the sedation protocol. Sleep deprivation before the EEG session is a standard, parents were instructed to put the children in bed $2 \mathrm{~h}$ later and wake the children $2 \mathrm{~h}$ earlier. Patients who were unable to cooperate for EEG setups were indicated for sedation. Under the old protocol, oral chloral hydrate $(50 \mathrm{mg} / \mathrm{kg}$, maximum $2 \mathrm{~g}$ ) in syrup form was given to patients requiring sedation, additional dose of chloral hydrate at $25 \mathrm{mg} / \mathrm{kg}$ would be given if the child was not sedated. In the new policy, syrup melatonin $(3 \mathrm{mg}$ for $=<5$ 
Table 1

EEG abnormalities [3].

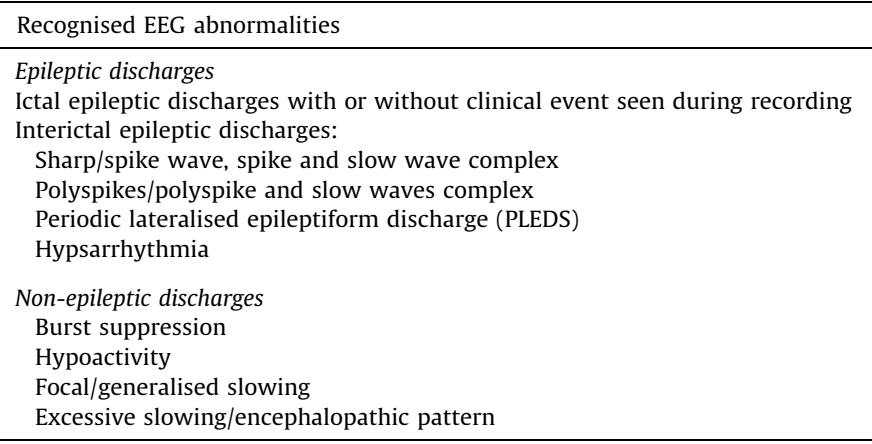

years old and $6 \mathrm{mg}$ for $>5$ years old) was provided as the primary sedative agent, if the child was not sedated, supplemented syrup chloral hydrate ( $50 \mathrm{mg} / \mathrm{kg}$, maximum $2 \mathrm{~g}$ ) was given. Patients who did not require sedation or with known hypersensitivity to the studied drugs were excluded. A total of 192 EEG recordings were included with 102 cases in the chloral hydrate group and 90 cases in the melatonin group. The respective drug was given orally $30-$ 45 min prior to the EEG studies by nursing staff. Regular body parameters including heart rate, respiratory rate and oxygen saturation were monitored before and after the investigation. The patients were discharged when they became fully awake and able to tolerate a meal.

All the EEGs were performed in a single neurophysiological laboratory with the same EEG machine (Biologic EEG) under the international $10-20$ system for 20-60 min. The EEGs were performed by the same electrophysiological technicians and were interpreted by the same paediatric neurologists. Asleep state, awake state, activations including photic stimulation and hyperventilation (if cooperation allowed) were included as the standard procedures. Data were collected from the hospital records and EEG request forms. The primary outcome is the effectiveness of melatonin as a sedation when compared with choral hydrate. The sedation was considered a failure if there was a need of additional doses of chloral hydrate in the chloral hydrate group or the need to provide chloral hydrate on top of melatonin in the melatonin group. Successful EEG was defined as being able to complete the whole session with acceptable motion artefacts for interpretation. The secondary outcome was the number of abnormal EEGs in each group and subgroup. The different EEG abnormalities are listed in Table 1. The data were compared with the Chi-squared test with Yates' continuity correction. A $p$ value of less than 0.05 was taken as statistically significant.
Table 2

Baseline characteristics.

\begin{tabular}{|c|c|c|c|}
\hline & $\begin{array}{l}\text { Chloral hydrate } \\
(n=102)\end{array}$ & $\begin{array}{l}\text { Melatonin } \\
(n=90)\end{array}$ & $p$ value \\
\hline Boys:girls & $59: 43$ & $57: 33$ & 0.53 \\
\hline Median age (year)/range & $\begin{array}{l}3.34 / 11 \text { days } \\
\text { to } 19 \text { years }\end{array}$ & $\begin{array}{l}3.65 / 6 \text { days } \\
\text { to } 22 \text { years }\end{array}$ & 0.10 \\
\hline History of febrile convulsion & 31 & 28 & 1.00 \\
\hline $\begin{array}{l}\text { Pre-existing diagnosis of } \\
\text { epilepsy }\end{array}$ & 47 & 50 & 0.93 \\
\hline Patients on anti-convulsant & 31 & 29 & 0.91 \\
\hline $\begin{array}{l}\text { Mentally retardation/ } \\
\text { developmental delay }\end{array}$ & 48 & 58 & 0.08 \\
\hline Cerebral palsy & 6 & 11 & 0.20 \\
\hline History of ADHD/autism & 18 & 14 & 0.85 \\
\hline History of prematurity & 8 & 13 & 0.22 \\
\hline
\end{tabular}

\section{Results}

Baseline characteristics, including age, sex and underlying conditions were similar in both groups, they are summarised in Table 2 . The background information about any previous history of febrile convulsion, whether or not the patient was on anti-epileptic agents, the pre-existed diagnosis of epilepsy, developmental delay or intellectual disability, cerebral palsy, hyperactivity and attention deficiency, autism and history of prematurity are of particular interests as these conditions associate with a increased potential of having epilepsy. Moreover, patients with underlying developmental problems are less readily to cooperate, we performed a subgroup analysis to assess the efficacy of the two studied sedative agents in sedating this group of patients.

For the primary outcome in assessing the effectiveness in sedating patients (Fig. 1), there is no statistically significant difference between the 2 studied agents, melatonin's sedation effect is comparable to chloral hydrate: 75 (83.33\%) in 90 cases of those in melatonin group succeeded in completing the EEGs without the need for additional drugs while in chloral hydrate group, $89(87.25 \%)$ in 102 cases succeeded $(p=0.57)$. A subgroup analysis regarding patients with chronic neurological problems as mentioned above was performed. Melatonin is equally effective among patients with chronic neurological problems (Fig. 2): 46 (79.31\%) in 58 cases of patients with developmental delay, mental retardation or cerebral palsy succeeded in completing the EEG in melatonin group and $40(83.33 \%)$ in 48 cases of patients with similar conditions succeeded in the chloral hydrate group $(p=0.78)$. There is no statistically significant difference. The cases which were successfully sedated by either melatonin or chloral hydrate alone were then included in the comparisons of the detection rate of abnormal EEGs. The cases failed to be sedated by any one of the above agents were excluded because they either
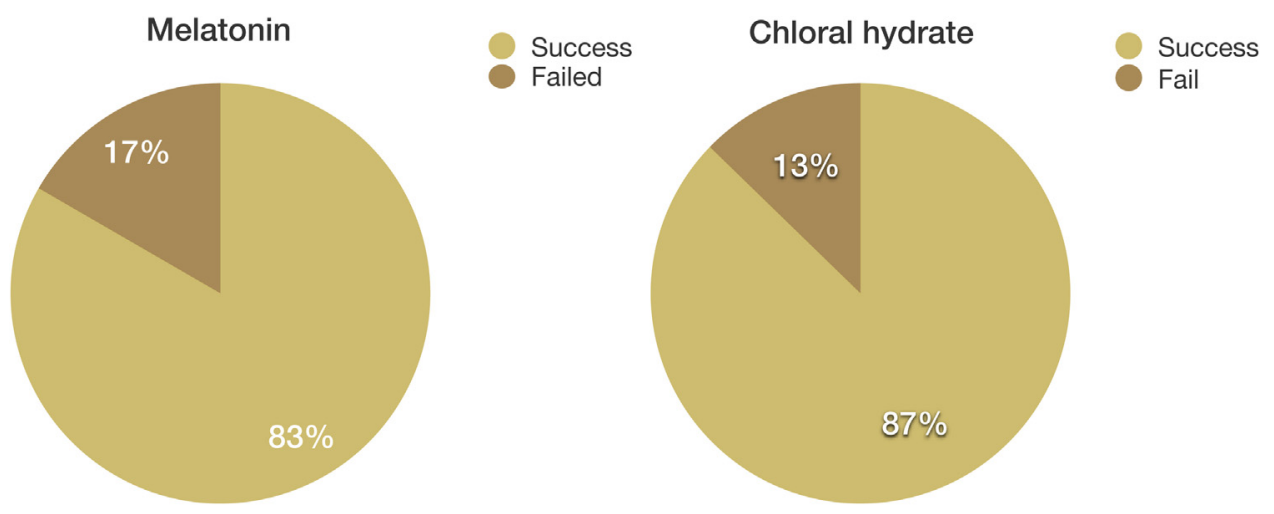

Fig. 1. Successful EEG $(p=0.57)$. 

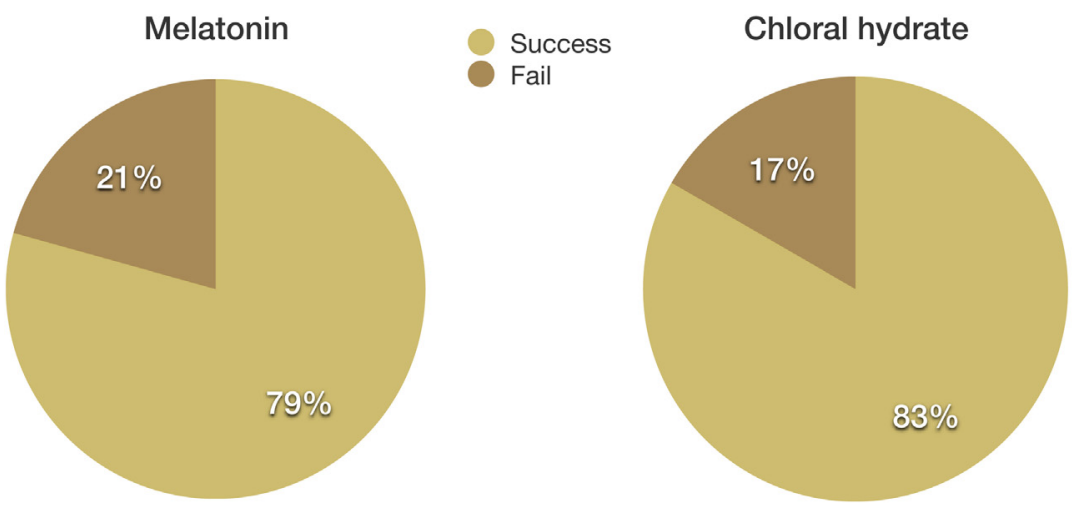

Success

Fail

Fig. 2. Successful EEG among patients with developmental delay/mental retardation/cerebral palsy $(p=0.78)$.

failed to complete the entire EEG studies or required additional dose or types of sedative agents to achieve satisfactory sedation, the additional sedative agents could exert their own anti-epileptic effects and affecting the outcome of the study. The baseline characteristics of the subgroups were compared again and there was no statistically difference between them.

We look at the detection rate of EEGs abnormalities in melatonin and chloral hydrate groups in different views. Abnormal EEGs were defined as EEGs that contained any one of the abnormal features listed in Table 1, no matter they were pure epileptiform discharges or background abnormalities, we identified 40 abnormal EEGs (52.56\%) in 75 cases in the melatonin group and $20(21.57 \%)$ in 89 cases in the chloral hydrate group (Fig. 3). The number of abnormal EEG identification is significantly higher in the melatonin group with $\mathrm{p}$ value $<0.05$. We further stratified the results by looking at EEGs with ictal or interictal epileptiform discharges only (Fig. 4). There were 38

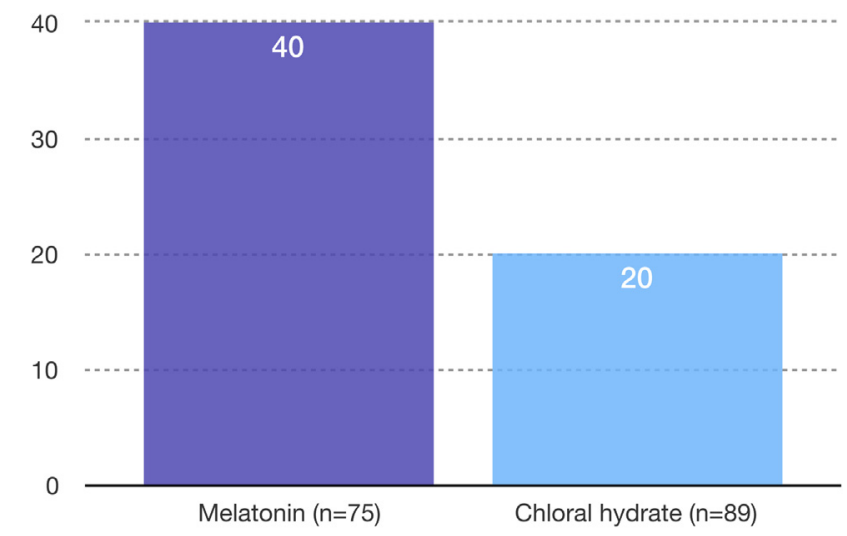

Fig. 3. Number of abnormal EEGs $(p<0.05)$.

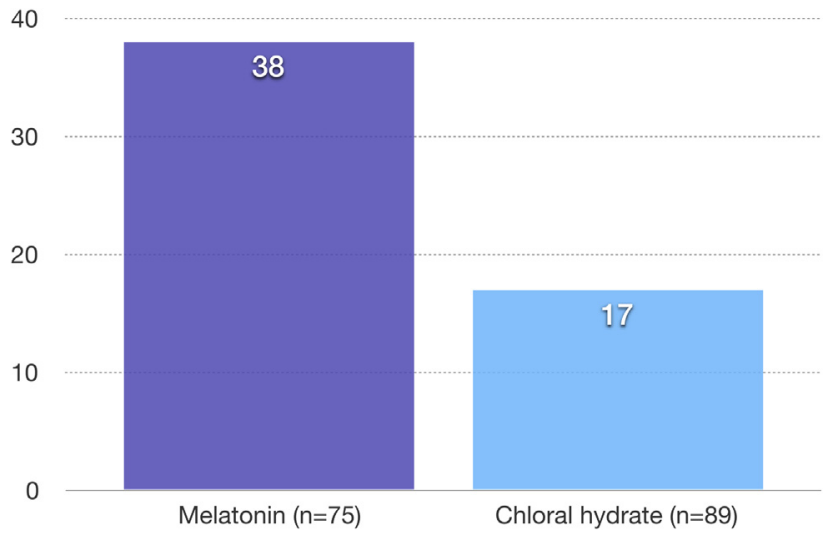

Fig. 4. Number of EEGs with epileptic discharges $(p<0.05)$.
(50.67\%) in 75 cases in the melatonin group and 17 (19.10\%) in 89 cases in the chloral hydrate group $(p<0.05)$, showing more epileptiform discharges are identified in the patients being sedated with melatonin.

We are also interested in whether patients with different underlying diagnosis would have different effects on the outcome, we performed comparison in patients who were already diagnosed epilepsy and those with the background history of mental retardation or intellectual disability as these patients are usually the focus of interest in patient care and have a higher chance of having abnormal EEGs. The number of abnormal EEGs detected among patients with the pre-existing diagnosis of epilepsy is significantly higher in the melatonin group: 29 cases $(76.32 \%)$ in 38 cases in the melatonin group versus $17(42.50 \%)$ in 40 cases in the chloral hydrate group $(p<0.05)$. Similar results were obtained in patients with mental retardation or intellectual disability where $31(67.39 \%)$ in 46 cases in the melatonin group were found to have abnormal EEGs compared to 14 (35\%) in 40 cases in chloral hydrate group $(p<0.05)$. The results come in line with our previous findings and suggested melatonin excels even when being compared in different subgroups. Regarding the side effect profile, both mediations and chloral hydrate give a good safety profile, there was no adverse effect documented during and after the study.

\section{Discussion}

Incidence of epilepsy is age related with a bimodal distribution, the highest rates occur in the youngest and eldest. ${ }^{4}$ The prevalence of epilepsy within the disability groups is also found to be higher than the general population, previous studies showed that by the age of 22 years, $22 \%$ of mentally retarded children had one episode of seizure and $15 \%$ had epilepsy. ${ }^{5}$ Children and patients with developmental delay or mental retardation are more prone to develop seizures but they are also the least likely to co-operate when performing EEGs. Properly performed interictal recordings could generally detect epileptiform discharges in half of the cases after the first unprovoked seizures. Combined awake and asleep EEGs can increase the yield to $80 \%$ as many epileptiform discharges appear in stage I or II sleep, such as benign focal epilepsy in childhood and some idiopathic generalised epilepsy. ${ }^{6}$ On the other hand, the chance of detecting photoparoxysmal response is higher when the patient is awake and can co-operate to instructions like eye opening or closing during photic stimulation. ${ }^{7}$ Therefore, both awake and asleep states are necessary in a properly performed EEG. The choice of an appropriate sedative agent is very important as we want the patients to attain adequate sleep stages but not getting to deep sedations and develop various serious side effects.

Chloral hydrate is absorbed from the gastrointestinal tract and is converted to its active metabolite trichloroethanol (TCE) by alcohol dehydrogenase. The onset of action is around 10-20 min 
and it reaches its maximum plasma concentration in 30-60 min. ${ }^{8}$ The exact mechanism of the sedative effect of TCE is still unknown. TCE undergoes glucuronidation in liver to form the inactive form of urochloralic acid or being oxidised to another inactive form namely the trichloroacetic acid. The main side effects are dizziness, paradoxical excitement, headache, nausea or vomiting and arrhythmia. Despite the side effects, chloral hydrate is generally well tolerated and has been widely used as a sedative agent in investigations like MRI or EEG for a long time. When being used as a sedation in EEG, the successful rate of sleep induction can reach $97 \%$ in previous study without significant side effects and our study shared similar results. ${ }^{9}$

Melatonin, chemically known as N-acetyl-5-methoxytryptamine, is a natural hormone synthesised in the pineal gland from tryptophan for regulation of the sleep-wake cycle. The secretion relates to our circadian cycle and is inhibited by the perception of light in the retina, the day time plasma level is low, it rises at night time and reaches its maximum plasma concentration at 3-4 am and fall again at dawn. Melatonin is oxidised to 6-OH-melatonin in liver and secreted in urine or faeces. Exogenous melatonin's sleep effect has been well studied. ${ }^{10}$ However, data on head-to-head comparison between melatonin and another sedative agent in EEGs are relatively sparse.

In our study, we showed that the sedation effect of melatonin is comparable to chloral hydrate with similar failure rate and there was no adverse effect reported. Our result is in line with previous studies where melatonin was found to be reliable in sleep induction when comparing with sleep deprivation. The studies also showed that it had shorter sleep latency, more predictable sleep onset time and most importantly, the sleep macroarchitecture was maintained. ${ }^{11,12}$ Another paper also indirectly reflected the usefulness of melatonin by concluding that they reduced the use of chloral hydrate to obtain a sleep EEG in children by $30 \%$ with the sequential administration of melatonin and hydroxyzine before chloral hydrate. ${ }^{13}$ The reported side effects of melatonin were minimal across all studies and there were even less behavioural disturbances when compared with sleep deprivation. ${ }^{14}$ All these features suggested melatonin is an appropriate choice of sedative agent in EEGs.

Our study found higher EEG abnormalities and epileptic discharge detections in the melatonin group. It is consistent with the result of Greta Gustafsson's study which demonstrated melatonin and sleep deprivation have the same occurrence rate of epileptiform discharges in EEGs in children ${ }^{15}$ and Mahmoud Rez Ashrafi's work on the comparison between melatonin and chloral hydrate as sedations in sleep EEGs. ${ }^{16}$ Anti-epilpetic effects of chloral hydrate have been suggested by case reports describing its use in the treatment of epilepsy. ${ }^{17,18}$ It was also found to reduce epileptic activities in EEGs in children given chloral hydrate as a sedation. ${ }^{19}$ However, there is absence of high level studies about the actual mechanisms of the anti-epileptic effects of chloral hydrate. We attempt to explain the phenomenon by the disturbance of the normal sleep architecture in patients being sedated with chloral hydrate. It is well known that epileptic discharges are more likely to occur in the drowsy state and light sleep stages instead of deeper slow-wave sleep stages. ${ }^{20}$ As TCE, the active metabolite of chloral hydrate, is closely related to ethanol, it should behave like ethanol and reduce sleep latency, decrease stage 1 sleep and increase slowwave sleep stages rendering a less favourable condition to detect epileptic discharges.

\section{Limitations}

Being a retrospective study is the main limitation of the current study. We tried to minimise the selection bias by changing the sedation protocol at a single time point and involved all patients undergoing routine EEGs. Separately, although the background characteristics of the patients are comparable in both groups, the differences in the underlying diseases, the seizure severities and the regime of anti-epileptic treatments still existed. These differences could affect the frequency of appearance of abnormal discharges on EEGs, it would be more representative if the patients can be stratified into very refined groups and be compared according to the individual diseases, however, this requires a way larger sample size to gather representable numbers of a particular type of disease or epileptic syndrome. In future studies, it would be valuable if we can also look into the medications' effects on the asleep stage of EEGs and assess the sleep architectures including the sleep onset latency, time of attaining different sleep stages and the maximum sleep stage achieved.

\section{Conclusion}

Melatonin is an effective and safe sedative agent in recording EEGs in children and has a higher detection rate of electrical abnormalities when compared to chloral hydrate.

\section{Conflicts of interest}

The authors have none to declare.

\section{References}

1. Slifera KJ, Avisc KT, Frutcheya RA. Behavioral intervention to increase compliance with electroencephalographic procedures in children with developmental disabilities. Epilepsy Behav. 2008;13:189-195.

2. Johnson K, Page A, Williams H, Wassemer E, Whitehouse W. The use of melatonin as an alternative to sedation in uncooperative children undergoing an MRI examination. Clin Radiol. 2002;57:502-506.

3. The International Federation of Societies for Electroencephalography and Clinical Neurophysiology, 1974. Electroencephalogr Clin Neurophysiol. 1974;37:521.

4. Panayiotopoulos CP. A Clinical Guide to Epileptic Syndromes and their Treatment (2nd ed.)

5. Goulden KJ, Shinnar S, Koller H, Katz M, Richardson SA. Epilepsy in children with mental retardation: a cohort study. Epilepsia. 1991;32:690-697.

6. Smith SJM. EEG in the diagnosis, classification, and management of patients with epilepsy. J Neurol Neurosurg Psychiatry. 2005;76:ii2-ii7.

7. Aminoff MJ. Aminoffs Electrodiagnosis in Clinical Neurology (6th ed.)

8. Pershad J, Palmisano P, Nichols M. Chloral hydrate: the good and the bad. Pediatr Emerg Care. 1999;15:432-435.

9. Jan MM, Aquino MF. The use of chloral hydrate in pediatric electroencephalography. Neurosciences. 2001;6:99-102.

10. Brzezinski A, Vangel MG, Wurtman RJ, et al. Effects of exogenous melatonin on sleep: a meta-analysis. Sleep Med Rev. 2005:9:41-50.

11. Wassmer E, Quinn E, Whitehouse W, Seri S. Melatonin as a sleep inductor for electroencephalogram recordings in children. Clin Neurophysiol. 2001;112: 683-685.

12. Eisermann M, Kaminska A, Berdougo B, Brunet ML. Melatonin: Experience in its use for recording sleep EEG in children and review of the literature. Neuropediatrics. 2010;41:163-166.

13. Dirani M, Nasreddine W, Melhem J, Arabi M, Beydoun A. Efficacy of the sequential administration of melatonin, hydroxyzine, and chloral hydrate for recording sleep EEGs in children. Clin EEG Neurosci. 2016;10:1-7.

14. Wassmer E, Carter PF, Quinn E, et al. Melatonin is useful for recording sleep EEG: prospective audit of outcome. Dev Med Child Neurol 2001:43:735-738.

15. Gustafsson G, Broström A, Ulander M, Vrethem M, Svanborg E. Occurrence of epileptiform discharges and sleep during EEG recordings in children after melatonin intake versus sleep-deprivation. Clin Neurophysiol. 2015;126:1493-1497.

16. Ashrafi MR, Mohammadi M, Tafarroji J, Shabanian R, Salamati P, Zamani GR Melatonin versus chloral hydrate for recording sleep EEG. Eur J Paediatr Neurol. 2010;14:235-238.

17. Krsek P, Sebronová V, Procházka T, Maulisová A, Komárek V. Successful treatment of Ohtahara syndrome with chloral hydrate. Pediatr Neurol. 2002;27:388-391.

18. Pranzatelli MR, Tate ED. Chloral hydrate for progressive myoclonus epilepsy: a new look at an old drug. Pediatr Neurol. 2001;25:385-389.

19. Thoresen M, Henriksen O, Wannag E, Laegreid L. Does a sedative dose of chloral hydrate modify the EEG of children with epilepsy? Electroencephalogr Clin Neurophysiol. 1997;102:152-157.

20. Dinner DS. Sleep and pediatric epilepsy. Cleve Clin J Med. 1989;56:234-239. 